\title{
Performance analysis of peak signal-to-noise ratio and multipath source routing using different denoising method
}

\author{
Kannadhasan Suriyan ${ }^{1}$, Nagarajan Ramaingam ${ }^{2}$, Sudarmani Rajagopal ${ }^{3}$, Jeevitha Sakkarai ${ }^{4}$, \\ Balakumar Asokan ${ }^{5}$, Manjunathan Alagarsamy ${ }^{6}$ \\ ${ }^{1}$ Department of Electronics and Communication Engineering, Cheran College of Engineering, Anna University, Tamilnadu, India \\ ${ }^{2}$ Department of Electrical and Electronics Engineering, Gnanamani College of Technology, Tamilnadu, India \\ ${ }^{3}$ School of Engineering, Avinashilingam Institute for Home Science and higher Education for Women, Coimbatore, Tamilnadu, India \\ ${ }^{4}$ Kalasalingam Institute of Technology, Krishnankoil, Tamilnadu, India \\ ${ }^{5}$ Department of Electronics and Communication Engineering, K.Ramakrishnan College of Engineering, Trichy, Tamilnadu, India \\ ${ }^{6}$ Department of Electronics and Communication Engineering, K.Ramakrishnan College of Technology, Trichy, Tamilnadu, India
}

\begin{tabular}{|c|c|}
\hline Article Info & ABSTRACT \\
\hline Article history: & The problem of denoising iris pictures for iris identification systems will be \\
\hline Received Aug 19, 2021 & Different salt and pepper extraction algorithms, as well as Gaussian and \\
\hline Revised Nov 12, 2021 & speckle noises, were used. Because diverse sounds decrease picture quality \\
\hline Accepted Dec 22, 2021 & $\begin{array}{l}\text { during image collection, noise reduction is even more important. To reduce } \\
\text { sounds like salt and pepper, Gaussian, and speckle, filtering (median, }\end{array}$ \\
\hline Keywords: & $\begin{array}{l}\text { wiener, bilateral, and Gaussian) and wavelet transform are utilised. Provide } \\
\text { better results as compared to other ways. A study of several efficiency }\end{array}$ \\
\hline $\begin{array}{l}\text { Discrete wavelet transform } \\
\text { Filter }\end{array}$ & $\begin{array}{l}\text { indicators such as peak signal-to-noise ratio (PSNR) and mean squared error } \\
\text { will be used to demonstrate the superiority of the proposed technique (MSE). }\end{array}$ \\
\hline
\end{tabular}

Mean squared error

Noise

Peak signal-to-noise ratio

This is an open access article under the $\underline{C C B Y-S A}$ license.

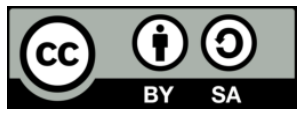

\section{Corresponding Author:}

Kannadhasan Suriyan

Department of Electronics and Communication Engineering, Cheran College of Engineering

Anna University, Karur, 600025 Tamilnadu, India

Email: Kannadhasan.ece@gmail.com

\section{INTRODUCTION}

Noise is unwelcome data that tarnishes a picture. The kind of noise present in the original picture plays an important role in the image filtering process. According to him, a decent picture denoising model should reduce noise as thoroughly as possible while maintaining edges. Denoising refers to the process of eliminating noise from a damaged picture. Denoising aims to reduce noise while preserving crucial visual properties. Amplifier noise (Gaussian noise), salt-and-pepper noise (impulse noise), poisson noise (shot noise), quantization noise (uniform noise), and speckle noise are some of the several forms of noise that may be seen in photographs (multiplicative noise). A linear low-pass filter may be sufficient if the input data includes a lot of noise but the magnitude is modest. A median filter, on the other hand, may be more suited if a picture has a little quantity of noise but a large magnitude. In each scenario, the filter process alters the image's overall frequency content. For denoising salt and pepper noise, Gaussian noise, and speckle noise, several filters such as median filter, ideal filter, butterworth filter, and bilateral filter were used. To evaluate the performance of filters, peak signal-to-noise ratio (PSNR) and mean squared error (MSE) are utilised. They determined that bilateral filters performed somewhat better than other filters in reducing Gaussian white noise based on the results. However, the denoised photos were still fuzzy. The median filter has a greater PSNR and better visual quality for retaining edges in pattern recognition. Based on decreased MSE values, 
the median filter approach outperformed the other three methods. It was able to recover a lot more information from the original picture and was a rather effective method of image denoising [1]-[5]. The wavelet transform is a useful tool for distinguishing between noisy and image signals. Because the wavelet transform is so good at compressing energy, the low coefficient is usually due to noise, whereas the high coefficient is due to important signal characteristics. These insignificant coefficients may be utilised as thresholds without affecting the images' essential characteristics. A filter pair is a wavelet transform. The filter bank is a technique for calculating the wavelet transforms by iteratively integrating and differentiating coefficients, one of which is a low pass filter (LPF) and the other a high pass filter (HPF). A model that filters a noisy image using a median filter and wavelet transform, then another median filter and wavelet transform. They got to the conclusion that if the image is impacted by Gaussian noise and speckle, the wavelet domain denoising approximation section should be filtered again using the median filter.

Individual wavelet domain and special domain filtering may provide superior results. For picture compression, they tested various wavelets [6]-[10]. The input image is initially classified as either genuine or fake in this example. After that, a thorough result was achieved employing several mother wavelets. For both natural and manufactured images, the Haar wavelet maintains more energy and has a greater number of zeros, according to the research. It shows that information is lost less often, resulting in increased efficiency. A wavelet model using Symlet wavelet and Wiener filters approach is used to decrease speckle noise in ultrasound images. This strategy surpasses earlier solutions by reducing mean square error. The wiener filter decreases the average mean square error in the inverse filtering and noise smoothing approach. The original signal is linearly estimated using Wiener filtering. In this model, the MSE value is 0.2359 , and the elapsed time is 5.669997 seconds. We studied several varieties of Coif let's wavelet family by compressing a fingerprint image using wavelet and wavelet packet transformations at three different threshold settings throughout the decomposition step. According to the findings of the experiment, the Coiflet5 is considerably stronger than other Coiflet-type wavelets for fingerprint photo compression for wavelet transform. The Bayesshrink technique and discrete wavelet transformations are used in this current methodology. Two noises, namely salt and pepper, and Gaussian noise, were effectively reduced from a single picture employing this strategy, and the results were found to be better than wiener and median filters due to a superior PSNR ratio and coefficient of correlation (COC) meaning. The suggested approach produces superior outcomes on ultrasound images [11]-[15].

\section{EXISTING METHOD}

An efficient approach for noise denoising addresses the problem of image denoising, which is still a challenging problem to solve in terms of devising algorithms to minimise the noise that is inherent in all visual images. This project provides a useful way for removing image noise. Some noise reduction techniques are well-known in the area of picture processing. The purpose of this study is to explain saltpepper, Gaussian, and Poisson sounds, as well as blends of two or three kinds of noises or noise in an image. Filtering techniques such as the median filter (MF), adaptive wiener filter (AWF), and adaptive median wiener filter (AMWF) are used for denoising. After that, a threshold-based denoising algorithm was built utilising a hard thresholding approach, and other filtering and thresholding techniques were added at the same time. The output is assessed using PSNR, MSE, and visual perception. The suggested technique delivers exceptional performance consistency for any kind of noise. Some linear and non-linear filtering, such as MF and AWF, is used on noisy data, and the effects are quantified using PSNR and visuality [16]-[20].

The median filter's value is denoised again using SWT hard thresholding if (PSNR) med > (PSNR) wien. The adaptive wiener filter's performance is denoised again by SWT rough thresholding if (PSNR) med $>$ (PSNR) wien. To remove the salt and pepper, Gaussian, and Poisson noise from the image and gather the denoised image. Examine the rituals as well. If the visual quality is poor, the process starts with a noisy file that is then denoised using the AMWF, which utilises a noise reduction approach that leverages varying window sizes. The window becomes bigger and bigger until the proper median value is determined. An adaptive filter estimates statistical information of every pixels' grey value, such as the local mean and local variance. SWT hard thresholding is then used to denoise the AMWF output. After that, the image is denoised. As a consequence, the image's noise combination (Gaussian + salt and pepper) and (Poisson and Gaussian) may be readily disregarded in this circumstance. [21]-[25], the suggested approach is utilised to reduce noise from a picture.

\section{PROPOSED DENOISING METHODOLOGY}

Wavelet-dependent denoising techniques (using soft or hard thresholding) give smoothness and improved edge retention, hence the suggested methodology leverages the wavelet domain as a major technique. A wavelet domain with a noise-reduction and efficiency-improving filter is shown in Figure 1. 
There are many different spatial filters and wavelets from which to pick. In the first level, the proposed approach uses a median filter, and in the second, a symlet wavelet. The discrete wavelet transform (DWT) is a method for encoding pictures at different resolutions as a series of approximations (low pass version) and information (high pass version). The image is low pass filtered to create an approximation image and high pass filtered to create detail images that represent the lost features while going from a higher to a lower resolution. A wavelet model using the Symlet wavelet and Wiener filters approach is used to reduce speckle noise in ultrasound images. This method surpasses prior methods by reducing the mean square error. Step 1: filter type 1 (spatial filter): non-linear filters like median (good for eliminating salt and pepper noise) and linear filters like Weiner (good for removing speckle noise) were used for filtering. Stage 2 (filter type 2) wavelet transform.

The wavelet transform (WT) is a signal and image processing method that has been effectively employed in a variety of scientific domains including signal processing, image compression, computer graphics, and pattern recognition. WT is a concise representation of image energy that aids in the determination of the threshold between noisy and essential image components. The continuous WT (CWT) approach extends the signal using basis functions formed by expanding, reducing, and shifting a single prototype function, known as the mother wavelet that was particularly chosen for the signal under consideration. The signal is decomposed into many scales with varying degrees of precision using this technique. The outcome of the transform appears as a time-scale representation because a scale parameter decreases or extends the mother wavelet in CWT. When considering the centre frequency of the mother wavelet, the scale parameter has an indirect relationship with frequency. A mother wavelet has a zero mean value, which necessitates the wavelet transform's transformation kernel to compactly support localization in time, allowing for the capturing of spikes that occur quickly. A wavelet expansion is a representation of a signal in terms of an orthogonal collection of real-values produced by performing appropriate transformations on the original wavelets. The primary distinction amongst mother wavelet functions like haar, daubechies, symlets, coiflets, and bi-orthogonal is the way their scaling signals and wavelets are defined.

Compression is a technique for lowering the amount of data needed to describe a particular image. Data redundancy is a major concern in digital picture compression. Techniques for data compression may be divided into two categories. Error free compression, also known as lossless compression, and error compression, sometimes known as lossy compression, are two types of compression. In this research, an experiment is presented to determine the optimal image compression strategy by computing and analysing PSNR values for different subimage sizes and quality parameters. PSNR is primarily intended for lossy compression methods; hence an experiment with different transform codes for lossy compression techniques is conducted. This software modified all transform coding approaches for this purpose. This research determines the PSNR value for each transform since several transform coding algorithms are utilised for digital picture compression. Because PSNR values are the performance metric of the transform coding schemes, they may be used to do a performance study. Digital filtering methods are used in DWT to produce a time-scale representation of the digital signal. The signal to be studied is routed through filters with various cutoff frequencies and scales. The discrete wavelet transform in two dimensions is basically a onedimensional analysis of a two-dimensional signal. It only analyses the rows and columns of a picture in a separable manner, and it only works on one dimension at a time. The picture is initially decomposed into areas using the Haar wavelet filter, with one region containing big numbers and the other region containing little numbers. This results in the creation of two new photos. $\mathrm{H}$ denotes rows and columns that have been evaluated using a high pass filter. Similarly, rows and columns evaluated using a low pass filter are labelled L. Following that, analysed filters are applied to the columns of each new picture, resulting in four distinct images known as subbands.

Wavelets are mathematical functions that split data into different frequency components and then explore each component with a resolution that is proportional to its size. They outperform ordinary Fourier algorithms in practical situations when the signal has discontinuities and abrupt spikes. The DWT is a method for expressing pictures at different resolutions as a series of approximations (low pass version) and information (high pass version). The image is low pass filtered to create an approximation image and high pass filtered to create detail images that represent the lost features while going from a higher to a lower resolution. The wavelet representation is then constructed using information coefficients at all resolutions and approximation coefficients at the lowest resolution. Linear and nonlinear filtering procedures are available in the wavelet domain. In recent years, there has been a lot of study on wavelet thresholding and threshold selection for signal de-noising, since wavelet gives an efficient basis for differentiating noisy signals from image signals. The mother wavelet selection and number of decomposition stages are the only differences between the de-noising approaches. In this scenario, the compression ratio was used to choose the mother wavelet for wavelet domain filtering. Symlet and coiflet have an $84.4013 \%$ compression ratio in natural 
pictures. The ratio is 83.8222 for both wavelets in manufactured images. As a consequence, denoising is done using the Symlet wavelet. HH, HL, LH, and LL are the four subbands of the image produced by filter 1 is shown in Table 1. The HH subband represents the image's diagonal details, the HL subband represents horizontal features, the LH subband represents vertical structures, and the LL subband represents the lowresolution residual, which is further separated at higher levels of decomposition as illustrated in Figure 2. Where $\mathrm{H}$ stands for high frequency bands, L stands for low frequency bands, and 1, 2, and 3 stands for decomposition levels. The comparison of the PSNR values for the different wavelets is shown in Figure 3 and Table 1. The peak signal-to-noise ratio (PSNR) is an engineering term that refers to the ratio of a signal's greatest achievable strength to the power of corrupting noise that affects the representation's quality. PSNR is measured in logarithmic decibel units because many signals have a large dynamic range.

PSNR is the most frequent metric for assessing the quality of lossy compression reconstruction. The signal represents the original data, while the noise represents the compression fault. PSNR is an approximation to human perception of picture reconstruction quality when comparing compression codecs. As a result, the greater the PSNR value, the better the picture reconstruction quality. The validity range of this measure must be carefully considered; it is definitively valid when used to compare results from the same codec and material. $\mathrm{C}$ was used to write the code.

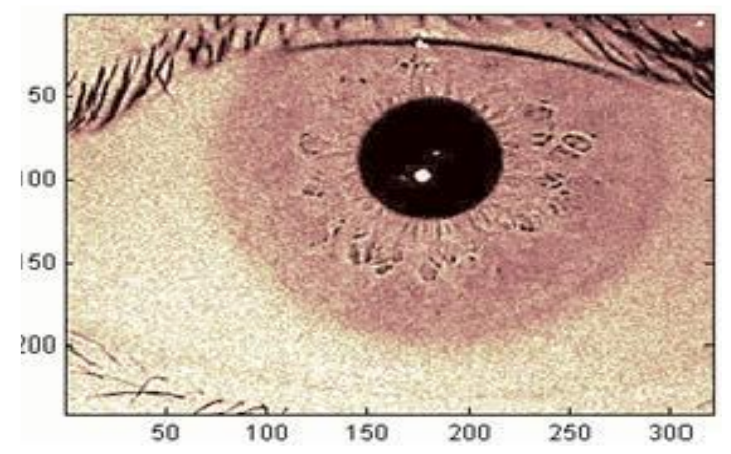

Figure 1. Original IRIS image

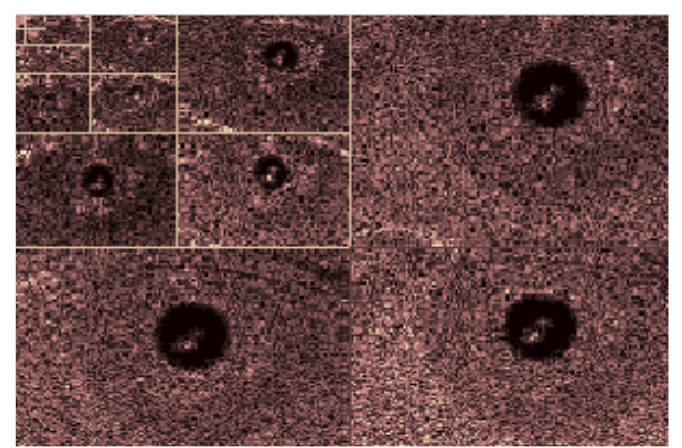

Figure 2. Decomposed image

Table 1. Comparison of PSNR values

\begin{tabular}{ccccc}
\hline Sl. No & Technique & PSNR salt and pepper noise & PSNR gaussian noise & PSNR speckle noise \\
\hline 1 & Median Filter & 27 & 24 & 27 \\
2 & COIFLET 1 & 21 & 27 & 30 \\
3 & SYMLET & 22 & 26 & 29 \\
4 & WEINER & 23 & 24 & 28 \\
5 & SYMLET+WEINER & 23 & 24 & 28 \\
6 & COIFLET+WEINER & 24 & 24 & 26 \\
7 & SYMLET+MEDIAN & 23 & 24 & 26 \\
8 & COIFLET+MEDIAN & 23 & 24 & \\
\hline
\end{tabular}




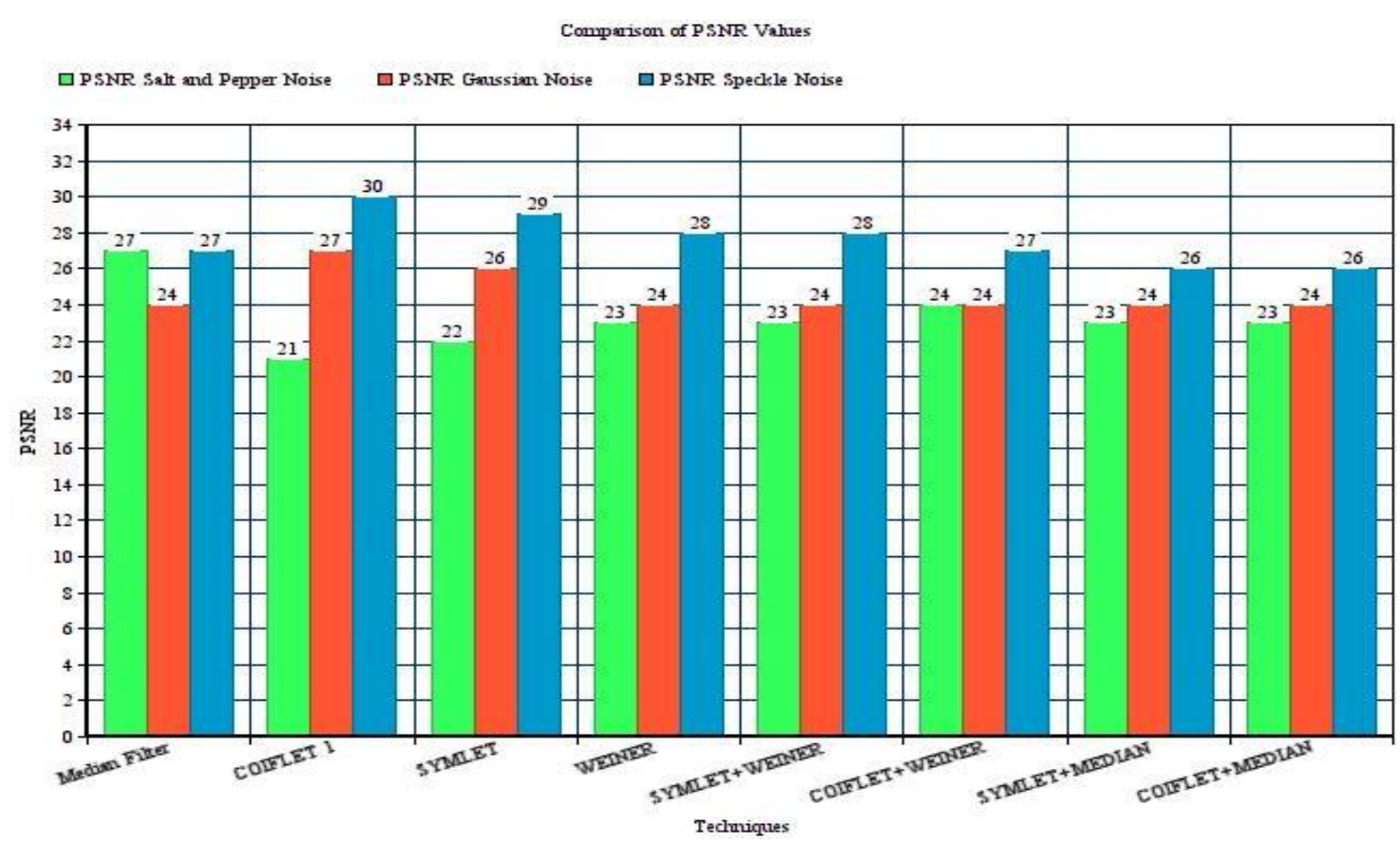

Figure 3. Comparison graph of PSNR values of different wavelets

\section{CONCLUSION}

The wavelet and median filters are used to solve the issue of denoising the iris picture for the Iris recognition system. Various denoising processes are used to remove salt and pepper, Gaussian, and speckle sounds, and the picture clarity is improved, allowing the Iris detection mechanism to work properly. About the fact that different noises such as salt and pepper, Gaussian, and speckle disturb iris images during image acquisition, the suggested technique, which combines the wavelet system with an adaptive Wiener approach dependent on statistics estimated from a local neighbourhood of each pixel, essentially removes the noises. Filtering approaches such as median, wiener, wavelet transform (symlet, coiflet, and biorthogonal) and principle part analysis are used to eliminate various noises. As opposed to other approaches, the proposed approach produces improved performance. Proposing alternative approaches would increase the PSNR performance of the proposed technique in the future. The proposed approach needs fewer memory, performs quickly, and is well suited for hardware execution with low power consumption and high processing performance.

\section{REFERENCES}

[1] P. Yu, J. Zhu, Z. Zhang, and Y. Han, “A Familial Cluster of Infection Associated With the 2019 Novel Coronavirus Indicating Possible Person-to-Person Transmission During the Incubation Period,',The Journal of infectious diseases, vol. 221, no. 11, pp. 1757-1761, May 2020, doi: 10.1093/infdis/jiaa077.

[2] S. Chavez, B. Long, A. Koyfman, and S. Y. Liang, "Coronavirus Disease (COVID-19): A primer for emergency physicians,' Am. J. Emerg. Med., vol. 44, pp. 220-229, June 2021, doi: 10.1016/j.ajem.2020.03.036.

[3] World Health Organization, "Coronavirus disease 2019 (COVID-19) Situation Report-142, 2020, [Online]. Available: https://www.who.int/docs/default-source/coronaviruse/situationreports/20200610-covid-19-sitrep-142.pdf?sfvrsn=180898cd_6 65 [cited 10 June 2020].

[4] Y. Bai et al., "Presumed Asymptomatic Carrier Transmission of COVID-19,'JAMA Network, vol. 323, no. 14, pp. 1406-1407, February 2020, doi: 10.1001/jama.2020.2565.

[5] Centres for Disease Control and Prevention, "Interim Infection Prevention and Control Recommendations for Patients with Suspected or Confirmed Coronavirus Disease 2019 (COVID-19) in Healthcare Settings," 2020 .[Online]. Available: https://www.cdc.gov/coronavirus/2019-ncov/hcp/infection-controlrecommendations.html. [cited 5 June 2020].

[6] R. Devarajapandian, M. Shanmuganantham and S. Kannadhasan, "Performance Improvement of Medical Images Using Wavelet Transformation," International Journal of Innovative Research in Science, Engineering and Technology (IJIRSET), vol. 10, no. 5, pp. 4452-4456, 2021, doi:10.15680/IJIRSET.2021.1005048.

[7] S. W. Sim, K. S. P. Moey, and N. C. Tan, "The use of facemasks to prevent respiratory infection: a literature review in the context of the Health Belief Model,'Singapore Medical Journal, vol. 55, no. 3, pp. 160-167, March 2014, doi: 10.11622/smedj.2014037.

[8] B. J. Cowling et al., "Facemasks and hand hygiene to prevent influenza transmission in households: a cluster randomized trial,", Annals of internal medicine, vol. 151, no. 7, pp. 437-446, October 2009, doi: 10.7326/0003-4819-151-7-200910060- 00142.

[9] S. M. Tracht, S. Y. D. Valle, and J. M. Hyman, "Mathematical modelling of the effectiveness of facemasks in reducing the spread 
of novel influenza A (H1N1),'PLoS One 5, p. e9018, February 2010, doi: 10.1371/journal.pone.0009018.

[10] T. Jefferson et al., "Physical interventions to interrupt or reduce the spread of respiratory viruses,' Cochrane Database System Rev., no. 7, p.CD006207, July 2011, doi: 10.1002/14651858.CD006207.pub4.

[11] K. M. Yorktown, M. Hake, D. R. Beukelman, and S. Fager, "Evidence for effectiveness of treatment of loudness, rate, or prosody in arthritis: A systematic review," Journal of Medical Speech-Language Pathology, vol. 15, no. 2, June 2007.

[12] A. E. Aron-son and D. Bless, "Clinical voice disorders,'”Thieme, 2009.

[13] D. C. Millay, J. C. Light, and R. W. Schloss, "The impact of augmentative and alternative communication intervention on the speech production of individuals with developmental disabilities: A research review," Journal of speech, Language, and Hearing Research, vol.49, no.2, April 2006, doi: 10.1044/1092-4388.

[14] N. S. Reese, "Auditory processing factors in language disorders: The view from Procrustes bed," Journal of Speech and Language Disorders, vol. 38, no.3, August 1973, doi: 10.1044/jshd.3803.304.

[15] A. P. Association, 'Diagnostic and Statistical Manual of Mental Disorders (DSM-5),'American Psychiatric Association, 2013.

[16] D. Mehta and K. Chauhan, “Compression of a filtered image using DCT-Technique,' International Journal of Electronics and Computer Science Engineering, vol. 2, pp. 919-926, 2012.

[17] P. Pawan, G. Manoj, S. Sumit, and A. K. Nagawat, "Image de-noising by various filters for different noise," International Journal of Computer Applications, vol. 9, no. 4, pp. 45-50, 2010, doi: 10.5120/1370-1846.

[18] I. Md. Fokhrul, H. A. M. Nazmul, B. A. Ara, C. S. Sharmin, and W. T. Binte, "Distribution of cancer patients and patterns of cancer treatment at Dhaka medical college hospital Bangladesh,' International Research Journal of Pharmacy, vol. 3, no. 4, pp. 157-161, 2012.

[19] Chaitanya P. M. and Sarada P. S., "Radiopharmacuticals for imaging of hypoxic tumors: A review,' International Research Journal of Pharmacy, vol. 3, no. 11, pp. 6-11, 2012.

[20] S. Shaikh, N. Akhter, and R. Manza, "Application of image processing techniques for characterization of skin cancer lesions using thermal images,' Indian Journal of Science and Technology, vol. 9, no. 15, pp. 1-7, 2016, doi: 10.17485/ijst/2016/v9i15/89794.

[21] R. H. Chan, Chung-WaHo, and M. Nikolova, "Salt-and-pepper noise removal by median-type noise detectors and detailpreserving regularization," in IEEE Transactions on Image Processing, vol. 14, no. 10, pp. 1479-1485, October 2005, doi: 10.1109/TIP.2005.852196

[22] C. A. Z. Barcelos and M. A. Batista, "Image restoration using digital inpainting and noise removal," Image and Vision Computing, vol. 25, no. 1, pp. 61-69, January 2007, doi: 10.1016/j.imavis.2005.12.008.

[23] C. Liu, R. Szeliski, S. B. Kang, C. L. Zitnick, and W. T. Freeman, "Automatic Estimation and Removal of Noise from a Single Image," in IEEE Transactions on Pattern Analysis and Machine Intelligence, vol. 30, no. 2, pp. 299-314, February 2008, doi: 10.1109/TPAMI.2007.1176.

[24] Z. Wang, A. C. Bovik, H. R. Sheikh, and E. P. Simoncelli, "Image quality assessment: from error visibility to structural similarity," in IEEE Transactions on Image Processing, vol. 13, no. 4, pp. 600-612, April 2004, doi: 10.1109/TIP.2003.819861.

[25] S. Grgi'c, M. Grgi'c, and M. Mrak, "Reliability of objective picture quality measures," Journal of Electrical Engineering, vol. 55, no. 1-2, pp. 3-10, 2004.

\section{BIOGRAPHIES OF AUTHORS}

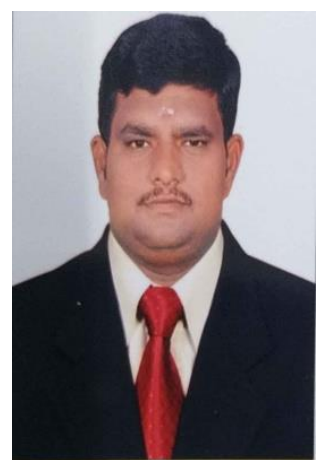

Kannadhasan Suriyan (D) $8 \mathrm{SC}$ S $\mathrm{P}$ is working as an Assistant Professor in the department of Electronics and Communication Engineering in Cheran College of Engineering, karur, Tamilnadu, India. He is currently doing research in the field of Smart Antenna for Anna University. He is ten years of teaching and research experience. He obtained his B.E in ECE from Sethu Institute of Technology, Kariapatti in 2009 and M.E in Communication Systems from Velammal College of Engineering and Technology, Madurai in 2013. He obtained his M.B.A in Human Resources Management from Tamilnadu Open University, Chennai. He obtained his PGVLSI in Post Graduate diploma in VLSI design from Annamalai University, Chidambaram in 2011 and PGDCA in Post Graduate diploma in Computer Applications from Tamil University in 2014. He obtained his PGDRD in Post Graduate diploma in Rural Development from Indira Gandhi National Open University in 2016. He has published around 18 papers in the reputed indexed international journals and more than 125 papers presented/published in national, international journal and conferences. Besides he has contributed a book chapter also. He also serves as a board member, reviewer, speaker, session chair, advisory and technical committee of various colleges and conferences. He is also to attend the various workshop, seminar, conferences, faculty development programme, STTP and Online courses. His areas of interest are smart antennas, digital signal processing, wireless communication, wireless networks, embedded system, network security, optical communication, microwave antennas, electromagnetic compatability and interference, wireless sensor networks, digital image processing, satellite communication, cognitive radio design and soft computing techniques. He is member of IEEE, ISTE, IEI, IETE, CSI, IAENG, SEEE, IEAE, INSC, IARDO, ISRPM, IACSIT, ICSES, SPG, SDIWC, IJSPR and EAI Community. He can be contacted at: kannadhasan.ece@gmail.com, and has website at: https://sites.google.com/view/kannadhasansuriyan-ece/ 

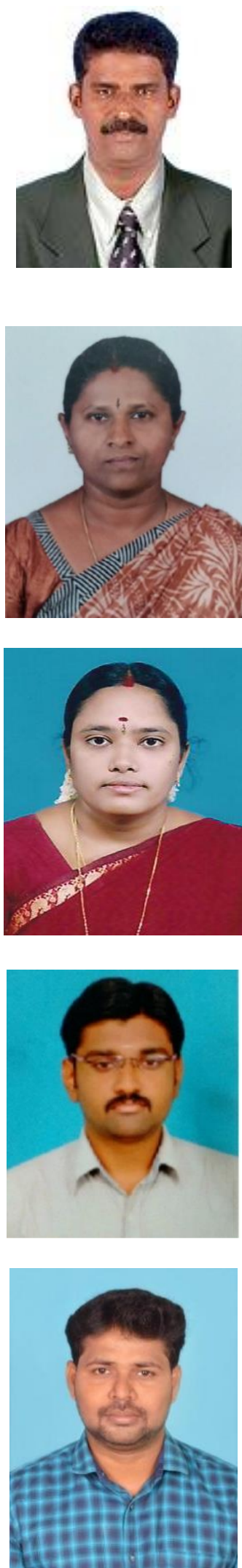

Dr. Nagarajan Ramalingam (D) $\mathrm{SD}$ SC $\mathrm{P}$ received his B.E. in Electrical and Electronics Engineering from Madurai Kamarajar University, Madurai, India, in 1997. He received his M.E. in Power Electronics and Drives from Anna University, Chennai, India, in 2008. He received his Ph.D in Electrical Engineering from Anna University, Chennai, India, in 2014. $\mathrm{He}$ has worked in the industry as an Electrical Engineer. He is currently working as Professor of Electrical and Electronics Engineering at Gnanamani College of Technology, Namakkal, Tamilnadu, India. He has published more than 70 papers in international journals and conferences. His research interest includes power electronics, power system, communication engineering, network security, soft computing techniques, cloud computing, big data analysis and renewable energy sources. He can be contacted at: krrajan71@gmail.com

Sudarmani Rajagopal (D) 8. SC P obtained her Bachelor's degree in Electronics and Communication Engineering from Kongu Engineering College, Perundurai, and her Master 's degree in Applied Electronics from Karunya Institute of Technology, Coimbatore. She obtained her doctoral degree from Anna University in the year 2014. She has 20 years of experience in teaching and now she is Associate Professor of the Department of Electronics and Communication Engineering, School of Engineering, Avinashilingam Institute, Coimbatore. Research interests are in the field of wireless sensor networks, signal processing and wireless communication. publications include 21 papers in international journals, 44 in conferences, 3 book chapters. Also presented one paper in TENCON IEEE International Conference at Indonesia in the year 2011. She can be contacted at: sudarmani_ece@avinuty.ac.in.

Jeevitha Sakkarai iD $8 \mathrm{SC}$ S received the M. Tech (Computer Applications) degree from Kalasalingam University in the year of 2010. She received the M.Sc (Computer Science) degree from SRM Arts \& Science, Chennai, Tamil Nadu, India, in 2004 and received the B.Sc (Computer Science) from Prof. Dhanabalan Arts \& Science College, Tamil Nadu, India in the year of 2002. She has been working as a Assistant Professor in the Department of Computer Science and Engineering, Kalasalingam Institute of Technology, Srivilliputhur, Tamil Nadu, since 2010. She is having more than 10 years' of experience in the field of teaching. Her research interests include image processing, data mining and big data analytics. She has published 13 papers in international journals and presented 9 papers in national and international journals. She can be contacted at jeevitha.ramkumar@gmail.com

Balakumar Asokan (iD 8. SC $\mathrm{P}$ received engineer degree in Electronics and Communication Engineering from St.Michael College Of Engineering and Technology in 2011. He received the Master degree in Communication Systems from B.S Abdhur Rahman Crescent University, Chennai in 2013. He is currently working as an Assistant Professor in the department of Electronics and Communication Engineering at K. Ramakrishnan College of Engineering, Trichy, India. His area of interest in cludes wireless sensor network, ad hoc networks, sensors and iterfacing networks and internet of things. He has published 6 articles in peer revieved journals and presented 3 papers in international conferences. He can be contacted at email: balakumar2712@gmail.com

Manjunathan Alagarsamy (iD) S8D SC received the Engineer degree in Electronics and Comunication Engineering from Dr. Navalar Nedunchezhiyan College of Engineering in 2010.He received the Master degree in Embedded System Technologies from Raja College of Engineering and Technology, Madurai, Tamilnadu, India in 2013.He is currently working as an Assistant Professor in the Department of Electronics and Communication Engineering at K. Ramakrishnan College of Technology, Trichy, India. His area of interests includes embedded systems, image processing, sensors and interfacing networks and internet of things. He has published 13 articles in peer reviewed International journals and presented 6 papers in international conferences. $\mathrm{He}$ can be contacted at email: manjunathankrct@gmail.com. 